$\mathrm{ABC}$ ) then the final committee list will rank the candidates in that order, $A B C$; if the five obey instructions by putting $B$ below $A$ (to get $\mathrm{CAB}$ ), the overall ranking will be $\mathrm{CAB}$; and if the hapless five simply switch $A$ and $B$ on their lists (to get ACB), then the final result is ACB. At this point it may seem a good idea to give up the Condorcet approach, and opt for the aggregate pointscore methods of Borda. But, as we have seen, these methods have their own faults, being among other things sensitive to the length of the list.

We are thus left with the conclusion that there is no foolproof way for a committee operating by majority rule to rank candidates on a list. Commonsense does, however, suggest some steps that can be useful. For one thing, time may be well spent in trying to argue out some partial or tentative consensus, rather than moving too crisply to one or other kind of vote. For another, it may be wise to keep lists as short as is consistent with their essential purpose, to reduce the scope for subtle (or even unconscious) manoeuvering. Failing all this, one can at least be alert to the above intricacies of the committee jungle. From that point of view, I was probably foolish to write this article.

Robert M. May is Class of 1877 Professor of Zoology at Princeton University, New Jersey 08544 .

\title{
Geomagnetic field
}

\section{New view on oscillations of magnetic shells}

\section{from Howard J. Singer}

VARIATIONS in the Earth's magnetic field with periods ranging from tens of seconds to around 10 minutes are classified as longperiod ultra-low-frequency (ULF) pulsations. In 1954, Dungey' suggested that these pulsations were standing hydromagnetic waves resonating on geomagnetic field lines, and numerous ground-based and spacecraft magnetometer observations have since supported and enriched the standing-wave model. Since 1977 , a new technique ${ }^{2}$, the Scandinavian twin auroral radar experiment (STARE), has made it possible to examine the electric-field signature of the longestperiod ULF waves ( $\mathrm{Pc} 5$ ) in the ionosphere at the foot of the field line. Among the STARE observations is a class of waves whose period increases with latitude for individual events ${ }^{3}$. These observations are important for understanding the source of the pulsations and the nature of the coupling of energy between individual oscillating shells of the geomagnetic field.

An important argument for the standing-wave model is that wave periods are consistent with the time it takes Alfvén waves to travel along field lines between ionospheric boundaries where the waves are reflected. The dependence of wave period on the background magnetic field and charged-particle mass density along the field line in the magnetosphere suggests the period should vary systematically with geomagnetic latitude. Indeed, groundbased observations have shown that although many pulsation events are essentially monochromatic over a wide range of latitude, statistically the longer-period events occur or have maximum amplitudes at higher latitudes. There are also some events which are composed of several discrete frequencies where the low frequencies dominate at high latitudes ${ }^{4}$. These two different types of observation have important implications for understanding the wave source. For example, in the first case the source is likely to be monochromatic and in the second case broad-band. It is therefore essential to know whether the groundbased observations are a reliable indicator of what is happening in the magnetosphere.

One difficulty in interpreting the ground-based observations is that the field observed on the ground is produced by currents in the ionosphere. Not only is wave energy lost in the ionosphere through Joule heating due to Pedersen currents, but the ionosphere acts as a spatial filter ${ }^{5}$ and severely attenuates short-scale signal variations of less than $120 \mathrm{~km}$. The STARE system is not subject to the ionospheric screening and in addition makes virtually simultaneous multiposition measurements in both latitude and longitude, making it possible to examine the spatial structure of the wave.

The STARE system ${ }^{6,7}$ can be used to measure the ionospheric electric field over a large area $(400 \times 400 \mathrm{~km})$ with high spatial $(20 \times 20 \mathrm{~km})$ and temporal $(20 \mathrm{~s})$ resolution. It consists of a pair of coherent pulse doppler radars located near Malvik, Norway and Hankasalmi, Finland. The principle of operation is based on the radar signal reflecting from electrostatic waves excited by instabilities in the auroral zone $E$ region. The doppler shift of the backscattered radar signal is a measure of the electron $E \mathrm{x}$ $B$ drift velocity which makes possible the determination of the amplitude and direction of the electric field in the overlapping region of the two radar beams. One limitation of the STARE system is a requirement that the electric field exceed a threshold value $\left(15-20 \mathrm{mV} \mathrm{m}^{-1}\right)$ to excite instabilities which reflect the radar pulse.

In a recent survey of STARE data ${ }^{3}, 49$ transient events, rarely visible for more than five cycles, were found where the individual pulsation events showed an increase in period with latitude. In one of the displayed events, the period changes from about $416 \mathrm{~s}$ to $500 \mathrm{~s}$ over about $3^{\circ}$ latitude at 1630 local time. These were toroidal eigenmode oscillations characterized by azimuthal oscillations of field lines in a shell around the Earth, although the longitudinal extent of the shell is not well established. At the time STARE observed these toroidal waves (magnetic east-west perturbation in space) a ground magnetometer at Tromso observed primarily a north-south perturbation. This result was consistent with theory ${ }^{5}$ that predicts a $90^{\circ}$ rotation of the magnetic field due to Hall currents in the intervening ionosphere. In addition, Poulter and Nielson ${ }^{3}$ have used the variation of the wave period with latitude to demonstrate a technique for determining the dependence of magnetospheric equatorial plasma density on radial distance in the vicinity of the STARE field lines.

The simultaneous occurrence of oscillating magnetic shells with different frequencies over a few degrees in latitude implies a broad-band or impulsive source for these waves. Such features have been observed before in space by radially moving spacecraft ${ }^{8}$, but only in special circumstances (for example, two closely spaced satellites) is it possible to separate spatial from temporal variations. The observation that field lines on adjacent magnetic shells can oscillate at different frequencies also suggests a weak coupling of energy between the field lines and should be of great interest to wave theorists and modellers.

The STARE observations have contributed to our understanding of ionospheric effects on magnetospheric ULF waves and will continue to do so. In the future, it should be possible to compare STARE measurements of pulsations with latitude-dependent frequencies with observations from a latitudinal chain of ground-based magnetometers. The comparison should explicitly determine the degree of screening by the ionosphere for this type of event.

Howard J. Singer is at the Plasmas, Particles \& Fields Branch, Space Physics Division, Air Force Geophysics Laboratory, Massachusetts 01731.

I. Dungey, J.W. Sci. Rep. 69 (Ionos. Res. Lab., PA State Univ. 1954); in Geophysics: The Earth's Environmen (eds DeWitt, Hieblot \& Lebeau) (Gordon \& Breach, New York, 1963).

Walker, A.D.M. Greenwald, R.A., Stuart, W.F.\& Green, C.A. Nature 273, 646 (1978).

3. Poulter, E.M.\& Nieisen, E. J. geophys. Res. 87, 10432 (1982).

4. Rostoker, G. \& Samson, J.C. J. geophys. Res. 77, 6249 (1972).

5. Hughes, W.J. \& Southwood, D.J. J. geophys. Res. 81, 3234 (1976).

6. Greenwald, R.A., Weiss, W., Nielsen, E. \& Thomsen, N.R. Radio Sci. 13, 1021 (1978).

7. Nielsen, E. IMS Source Book (eds Russell, C.T. \& Southwood, D.J.) 213 (Am. geophys. Un., Washington DC, 1982).

8. Singer, H.J., Russell, C.T., Kivelson, M.G., Fritz, T.A. \& Lennarisson, W. Geophys. Res. Lett. 6. 889 (1979). 